\title{
Relationship between changes of electrocardiogram indexes in chronic heart failure with arrhythmia and serum PIIINP and BNP
}

\author{
YUE WANG $^{1}$ and XIAOKE MA ${ }^{2}$ \\ ${ }^{1}$ Electrocardiogram Room, Yantai Yuhuangding Hospital; ${ }^{2}$ Departnent of Laboratory Medicine, \\ Yantai Hospital of Traditional Chinese Medicine, Yantai, Shandong 264000, P.R. China
}

Received September 23, 2019; Accepted November 20, 2019

DOI: $10.3892 /$ etm.2019.8269

\begin{abstract}
Relationship between changes of electrocardiogram (ECG) indexes in chronic heart failure (CHF) with arrhythmia and serum type III procollagen amino-terminal peptide (PIIINP) and brain natriuretic peptide (BNP) were evaluated. From December 2017 to December 2018, 101 patients with heart failure (HF) were collected. Among them, 48 patients with HF and slow arrhythmia were in group A, and 53 cases of HF with non-slow arrhythmia were sin group B, including 33 males and 20 females. BNP was detected by chemiluminescence and PIIINP was detected by immunoassay. The changes of ECG indexes in the two groups, the correlation between serum PIIINP and BNP and NYHA classification of cardiac function, and the correlation between ECG indexes and PIIINP and BNP were detected. ROC curve analysis of BNP and PIIINP in the diagnosis of slow HF was carried out. Serum PIIINP and BNP in group A were significantly higher than those in group $\mathrm{B}(\mathrm{P}<0.05)$. The levels of PIIINP and BNP in serum of NYHA patients with different cardiac functions, and those in serum of patients with class III were significantly higher than those of group II $(\mathrm{P}<0.05)$, while significantly lower than those of group IV $(\mathrm{P}<0.05)$. The heart rate and Q-T interval in group A were significantly higher than those in group $\mathrm{B}(\mathrm{P}<0.05)$. The $\mathrm{P}-\mathrm{R}$ interval and $\mathrm{QES}$ wave group in group A were significantly lower than those in group B $(\mathrm{P}<0.05)$. BNP had a positive correlation with $\mathrm{Hr}$ and $\mathrm{G}-\mathrm{T}$, and was negatively correlated with P-R and QRS; PIIINP was positively correlated with $\mathrm{Hr}$ and G-T, and had a negative correlation with P-R, QRS and BNP; PIIINP had positive correlation with NYHA; ECG indexes were correlated with
\end{abstract}

Correspondence to: Dr Xiaoke Ma, Departnent of Laboratory Medicine, Yantai Hospital of Traditional Chinese Medicine, 39 Xingfu Road, Yantai, Shandong 264000, P.R. China

E-mail: kod93s@163.com

Key words: chronic heart failure, electrocardiogram indexes, PIIINP, BNP
BNP and PIIINP, and had diagnostic value for CHF. Using ECG indexes to predict BNP and PIIINP levels was conducive to the diagnosis of CHF.

\section{Introduction}

Chronic heart failure (CHF) (1) is a group of clinical syndromes caused by changes in cardiac structure and function caused by various reasons, leading to the reduction of left ventricular filling and ejection fraction. It is the final stage of development of various cardiovascular diseases and is also the main cause of death (2). Heart failure (HF) refers to cardiac circulatory disorder syndrome caused by failing to fully discharge venous return volume out of the heart due to dysfunction of cardiac systolic and/or diastolic function, resulting in blood deposition in venous system and insufficient blood perfusion in arterial system. Such disorder syndrome is mainly manifested as pulmonary congestion and vena cava congestion (3). HF is not an independent disease, but the final stage of the development of heart diseases, most of which begin with left HF (4). Therefore, it is of great significance to find the disease in time and control and treat it to remission.

Studies have shown that patients with CHF have pathophysiological changes such as myocardial electrical remodeling, which can be characterized by cardiac rhythm. Therefore, electrocardiogram (ECG) of patients with CHF may have special ECG manifestations (5). Some studies have shown that myocardial interstitial collagen deposition in patients with CHF and arrhythmia can affect the maintenance of ventricular structure and cardiac function (6). Previous studies have shown that type III procollagen amino-terminal peptide (PIIINP) is a metabolic product during myocardial collagen synthesis, which can better reflect the index of collagen fiber formation in vivo (7). Brain natriuretic peptide (BNP) is mainly synthesized in ventricular myocytes and secreted by the left ventricle. It flows back into the small vein to the interventricular septum vein and enters the circulation through the coronary sinus $(8,9)$. Ventricular wall tension, pressure overload, myocardial ischemia, necrosis and injury can stimulate the synthesis and release of BNP. BNP is a sensitive index reflecting ventricular function and load, and is widely used in evaluating cardiac function (10). 
Table I. General data.

\begin{tabular}{lcccc}
\hline Factor & Group A $(\mathrm{n}=48)$ & Group B $(\mathrm{n}=53)$ & $\chi^{2} / \mathrm{t}$ & P-value \\
\hline Sex & & & 1.958 & 0.1439 \\
Male & 31 & 33 & & \\
Female & 16 & 20 & 1.743 & 0.814 \\
Age & $57.6 \pm 3.7$ & $59.2 \pm 4.1$ & 0.5912 & 0.3541 \\
Course of disease & $1.75 \pm 0.07$ & $1.89 \pm 0.09$ & 1.740 & 0.4583 \\
Coronary heart disease & 23 & 24 & 1.348 & 0.3674 \\
Hypertension & 13 & 15 & 1.181 & 0.4931 \\
Rheumatic heart disease & 8 & 5 & 1.331 & 0.2405 \\
Dilated cardiomyopathy & 4 & 9 & & 0.4710 \\
NYHA & & & 0.5188 & 0.7813 \\
Class II & 14 & 16 & 0.001 & 0.5960 \\
Class III & 25 & 27 & 2.782 & 0.9995 \\
Class IV & 9 & 10 & 7.509 & 0.5949 \\
Lown class III & 24 & 24 & 0.1053 & 0.0234 \\
Lown class IV & 17 & 18 & & 0.9487 \\
Lown class V & 7 & 11 & & \\
\hline
\end{tabular}

Therefore, the purpose of this investigation was to monitor the condition of patients with CHF and arrhythmia by observing the changes of ECG indexes, and to study the relationship between ECG indexes and serum PIIINP and BNP.

\section{Patients and methods}

General information. From December 2017 to December 2018, 101 patients with HF were collected. Among them, 48 patients with HF and slow arrhythmia were in group A, including 31 males and 16 females, with an average age of 57.6 \pm 3.7 years and a course of disease of $1.75 \pm 0.07$ years, while 53 cases of those with HF and non-slow arrhythmia were in group B, including 33 males and 20 females, with an average age of $59.2 \pm 4.1$ years and course of disease of $1.89 \pm 0.09$ years Further data are shown in Table I. There was no statistical significance between the two groups.

The study was approved by the Ethics Committee of Yantai Yuhuangding Hospital (Yantai, China). Signed informed consents were obtained from the patients and/or the guardians.

Exclusion and inclusion criteria. Inclusion criteria (1) were CHF B with arrhythmia confirmed by ECG and echocardiography, and left ventricular ejection fraction $\leq 40 \%$ (11). Exclusion criteria were: i) thyroid and lung diseases; ii) sick sinus syndrome patients; iii) those who did not wish to be included in this study.

\section{Methods and detection indexes}

Specimen collection. Altogether 2-3 $\mathrm{ml}$ venous blood of all the selected patients were taken on admission, and the next day on an empty stomach, and the relevant biochemical indexes were checked.

BNP test. Fasting venous blood of patients was collected, $15 \%$ EDTA was added into anticoagulation test tube, and centrifuged at $1,509.3 \times \mathrm{g}$ at $4^{\circ} \mathrm{C}$ for $10 \mathrm{~min}$ with an effective centrifugal radius of $15 \mathrm{~cm}$. The blood was detected by chemiluminescence using Pulangpuzs-300 series automatic biochemical analyzer (Hitachi 7180 type).

PIIINP detection. Fasting venous blood of patients was collected, $15 \%$ EDTA was added to anticoagulation test tube, centrifuged at $1,509.3 \mathrm{x}$ g at $4^{\circ} \mathrm{C}$ for $10 \mathrm{~min}$ with an effective centrifugal radius of $15 \mathrm{~cm}$, and the separated serum was stored in a low temperature refrigerator at $-20^{\circ} \mathrm{C}$ for later use. Balanced radioimmunoassay was used for determination, and radioimmunoassay reagent was provided by Biotechnology Center of Hunan Pharmaceutical Research Institute.

Detection of ECG index changes in the two groups. After admission, the ECG changes of the two groups were observed, including heart rate, Q-T interval, P-R interval and QRS wave group, and recorded.

Correlation between serum PIIINP, BNP and NYHA classification of cardiac function (12). Patients with different NYHA classification of cardiac function were grouped, and the expression levels of PIIINP and BNP in the patients were detected. Through Pearson correlation factor analysis, the correlation between serum PIIINP, BNP and NYHA classification of cardiac function was analyzed.

Correlation between ECG indexes and PIIINP and BNP. Through Pearson correlation factor analysis, the correlation between ECG indexes and PIIINP and BNP expression level was analyzed.

ROC curve analysis (13). The receiver operating characteristic curve (ROC) was used to analyze whether serum PIIINP and BNP had diagnostic value in CHF with arrhythmia. 
A

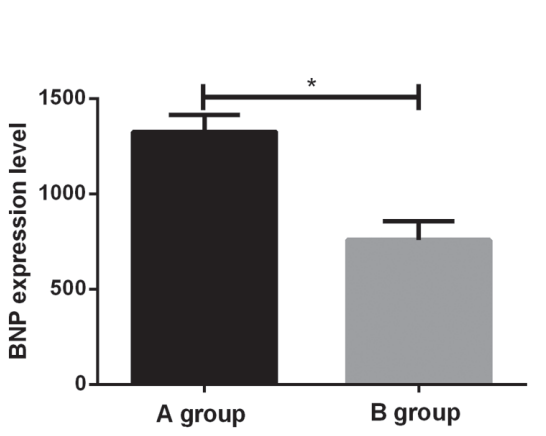

C

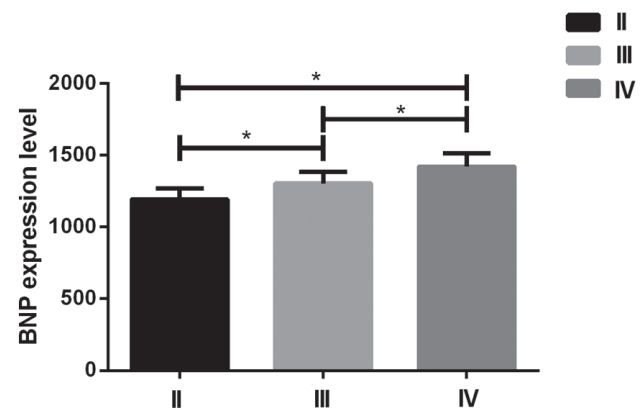

B

A group

$B$ group

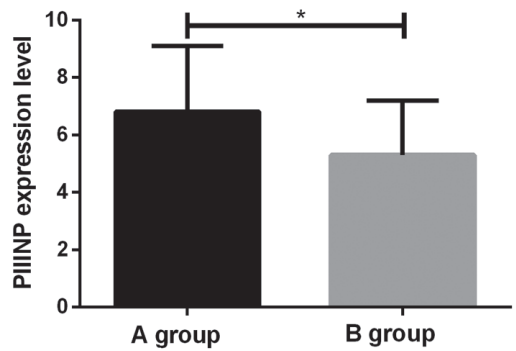

A group

$B$ group

D

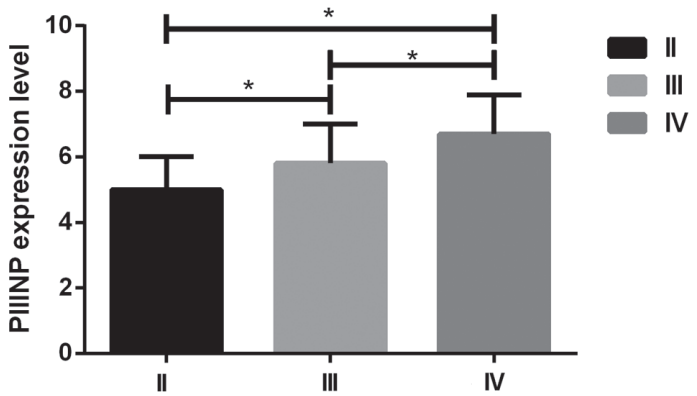

Figure 1. Contents of serum PIIINP and BNP of patients in the two groups. (A) BNP level in serum of group A was significantly higher than that in group B. (B) PIIINP level in serum of group A was significantly higher than that of group B. (C) BNP level in serum of patients with class III was significantly higher than that in group II and significantly lower than that in group IV. (D) PIIINP level in serum of patients with class III was significantly higher than that in group II and significantly lower than that in group IV. ${ }^{*} \mathrm{P}<0.05$. PIIINP, type III procollagen amino-terminal peptide; BNP, brain natriuretic peptide.

Statistical methods. This study used SPSS18.0 software (Bizinsight (Beijing) Information Technology Co., Ltd.) to carry out statistical analysis on the data. GraphPad Prism 6 software was used to draw the illustrations in this study. Chi-square test was used to compare the counting data, and mean \pm standard deviation to express the measurement data. t-test was emplyed to analyze the two groups, and variance analysis to compare the multiple groups, and Pearson correlation analysis was used to analyze the relationship between variables. $\mathrm{P}<0.05$ was considered to indicate a statistically significant difference.

\section{Results}

Levels of serum PIIINP and BNP of patients in the two groups. The content of serum PIIINP and BNP of patients in the two groups showed that the serum PIIINP and BNP in group A were significantly higher than those in group $\mathrm{B}(\mathrm{P}<0.05)$. The levels of PIIINP and BNP in serum of NYHA patients with different cardiac functions, and those in serum of patients with class III were significantly higher than those of group II $(\mathrm{P}<0.05)$, while significantly lower than those of group IV $(\mathrm{P}<0.05)$ (Fig. 1)

Changes of ECG indexes in the two groups. The ECG indexes such as heart rate, Q-T interval, P-R interval and QRS wave of patients in the two groups were examined. The results revealed that the heart rate and Q-T interval of group A were
Table II. ROC curve analysis.

\begin{tabular}{lcccc}
\hline Indicators & AUC & SE & $95 \%$ CI & P-value \\
\hline BNP & 0.8829 & 0.03548 & $0.8133-0.9524$ & $<0.001$ \\
PIIINP & 0.6340 & 0.05561 & $0.5250-0.7431$ & $<0.001$ \\
\hline
\end{tabular}

BNP, brain natriuretic peptide; PIIINP, type III procollagen amino-terminal peptide.

significantly higher than those of group $\mathrm{B}(\mathrm{P}<0.05)$, and the $\mathrm{P}-\mathrm{R}$ interval and QES wave in group A were significantly lower than those in group $\mathrm{B}(\mathrm{P}<0.05)$ (Fig. 2).

Correlation analysis. Pearson correlation analysis indicated that BNP was correlated with Hr, G-T, P-R and QRS, positively correlated with $\mathrm{Hr}$ and G-T $(\mathrm{r}=0.7749$, $\mathrm{r}=0.6743)$, and was negatively correlated with P-R and QRS ( $\mathrm{r}=-0.6684$, $\mathrm{r}=-0.6811$ ). PIIINP was positively correlated with $\mathrm{Hr}, \mathrm{G}-\mathrm{T}$, $\mathrm{P}-\mathrm{R}$, and QRS ( $\mathrm{r}=0.5509, \mathrm{r}=0.5635)$, and was negatively correlated with P-R and QRS ( $\mathrm{r}=-0.5730, \mathrm{r}=-0.7707)$. BNP, PIIINP and NYHA had positive correlation $(r=0.5804, r=0.5813)$ (Figs. 3 and 4).

ROC curve analysis. ROC curve analysis represented that ACU of BNP in diagnosing patients with $\mathrm{CHF}$ was 0.8829 , the 
A

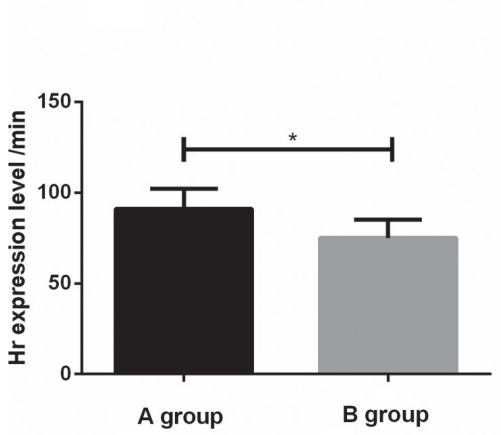

C

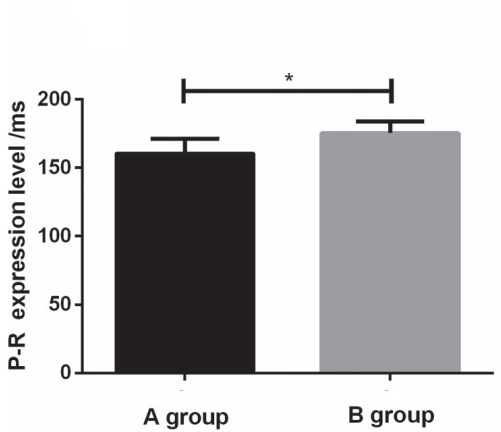

B

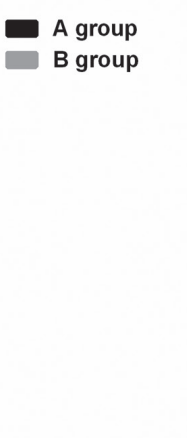

A group

B group

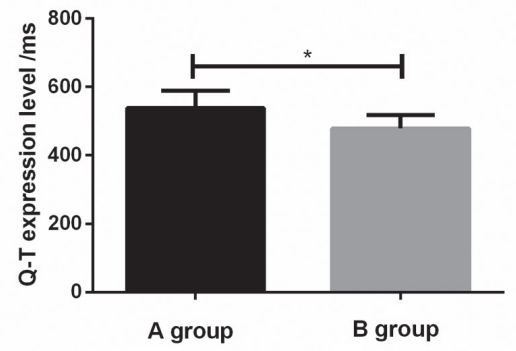

D

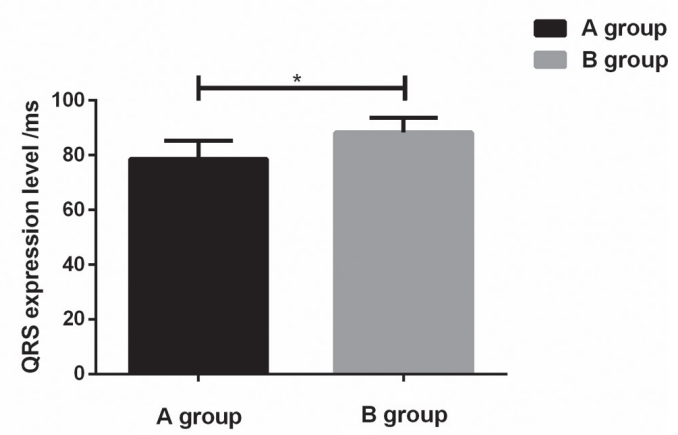

A group

B group

Figure 2. Changes of ECG indexes in the two groups. (A) ECG Hr in group A was significantly higher than that in group B. (B) ECG Q-T in group A was significantly higher than that in group B. (C) ECG P-R in group A was significantly lower than that in group B. (D) ECG QRS in group A was significantly lower than that in group $\mathrm{B}$. "P $<0.05$. ECG, electrocardiogram.

A

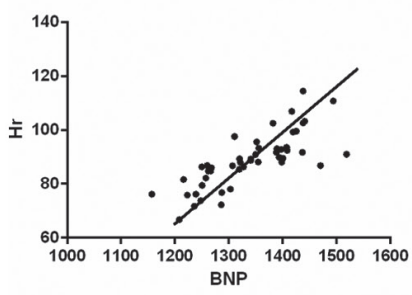

$\mathbf{E}$

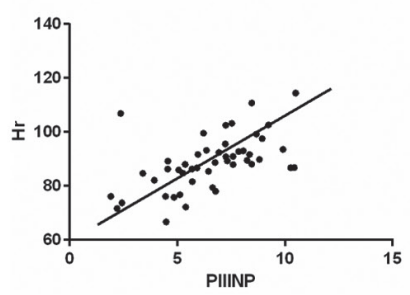

B

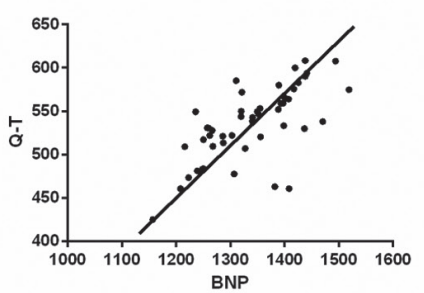

$\mathbf{F}$

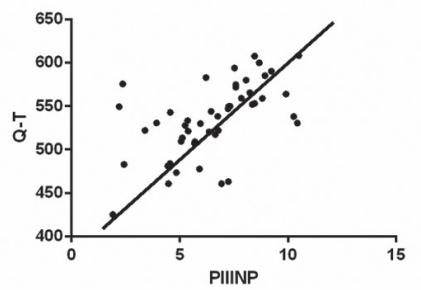

C

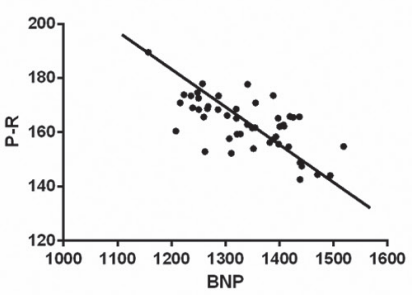

G

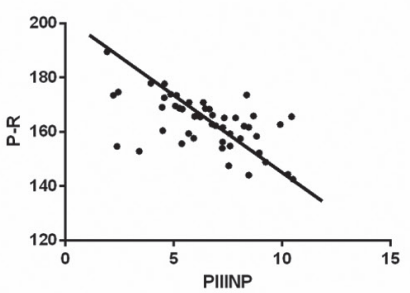

Figure 3. Correlation analysis of BNP and PIIINP with Hr, G-T, P-R and QRS (A) BNP had a positive correlation with Hr (r=0.7749). (B) BNP had a positive correlation with G-T ( $\mathrm{r}=0.6743)$. (C) BNP was negatively correlated with P-R ( $\mathrm{r}=-0.6684)$. (D) BNP was negatively correlated with QRS ( $\mathrm{r}=-0.6811)$. (E) PIIINP had a positive correlation with $\mathrm{Hr}(\mathrm{r}=0.5509)$. (F) PIIINP had a positive correlation with $\mathrm{G}-\mathrm{T}(\mathrm{r}=0.5635)$. (G) PIIINP had a negative correlation with P-R $(\mathrm{r}=-0.5730)$. (H) PIIINP had a negative correlation with QRS ( $\mathrm{r}=-0.7707)$. The correlation analysis yielded significant results $(\mathrm{P}<0.05)$. BNP, brain natriuretic peptide.

sensitivity was $69.51 \%$, and the specificity was $81.61 \%$; AUC of PIIINP in diagnosing patients with CHF was 0.6340 , the sensitivity was $73.64 \%$, and the specificity was $52.71 \%$. (Table II and Fig. 5).

\section{Discussion}

HF usually refers to the deterioration of myocardial contractility, which leads to insufficient output to meet the needs of 
A

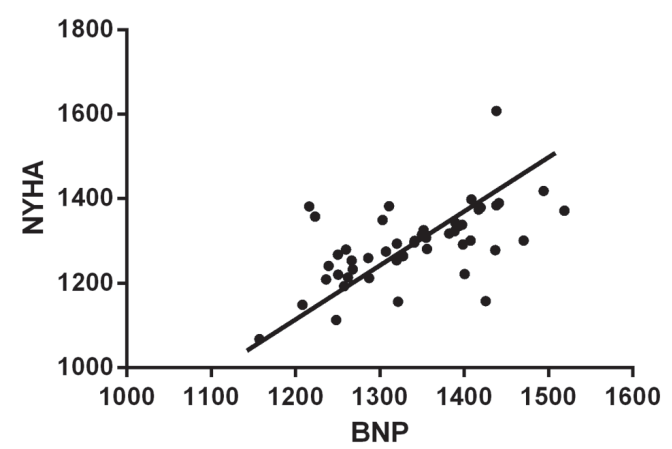

B

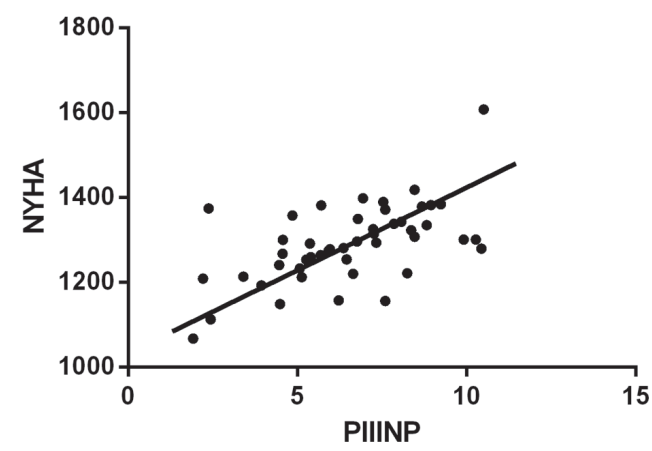

Figure 4. Correlation analysis of BNP, PIIINP and NYHA. (A) BNP had positive correlation with NYHA ( $\mathrm{r}=0.5804)$. (B) PIIINP had a positive correlation with NYHA $(r=0.5813)$. The correlation analysis yielded significant results $(\mathrm{P}<0.05)$. BNP, brain natriuretic peptide.

A

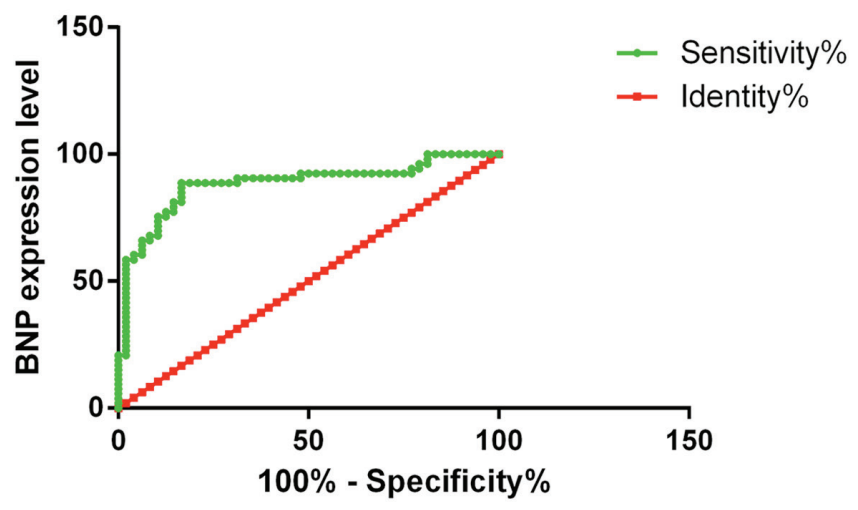

B

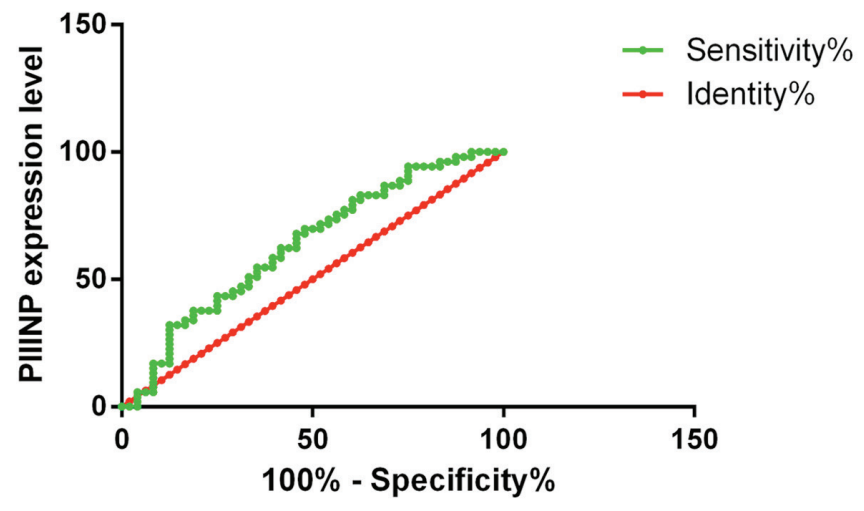

Figure 5. ROC curve analysis. (A) ACU of BNP in diagnosing patients with CHF was 0.8829 , the sensitivity was $69.51 \%$ and the specificity was $81.61 \%$. (B) AUC of PIIINP in diagnosing patients with CHF was 0.6340 , the sensitivity was $73.64 \%$, and the specificity was $52.71 \%$. BNP, brain natriuretic peptide; $\mathrm{CHF}$, chronic heart failure.

collective metabolism, affecting the perfusion of organs and tissues, and is often accompanied by passive hemorrhage of systemic circulation and pulmonary circulation. CHF is mainly caused by physiological changes, and the pathogenesis of ventricular arrhythmia is mainly abnormal depolarization activity, increased self-discipline and conduction reversion. When CHF progresses to hypofilling function or ventricular pumping, or ventricular tachycardia and ventricular arrhythmia is prematurely triggered, leading to arrhythmia, and in serious cases, ventricular fibrillation will even lead to death (14). Antiarrhythmic drugs are commonly used in clinical treatment of $\mathrm{CHF}$ with ventricular arrhythmia, which can effectively improve clinical symptoms and control arrhythmia, but the treatment effect is slow.

Collagen in myocardial interstitium is mostly type III collagen. Collagen, like other proteins, is transcribed into mRNA in cells via DNA and then translated and expressed as procollagen $\alpha$ peptide. The amino end and carboxyl end of procollagen each have a non-collagen terminal peptide. After hydroxylation, procollagen molecules form a triple helix, which is secreted by cardiac fibroblasts after saccharification (15). Therefore, PIIINP can accurately reflect the active process of collagen synthesis in cardiac tissue. BNP is mainly produced in ventricles, and the release of BNP increases with the increase of ventricular wall tension and load in HF. At present, many studies have proved that BNP plays an important role in the diagnosis and risk degree of $\operatorname{HF}(16,17)$. It is also believed that BNP can be used as the mortality indicator of patients with HF in hospital and at 6 months and one year after discharge (18), but there is a lack of research on arrhythmia (19). This study found that the concentration of BNP in plasma of ventricular arrhythmia group was significantly higher than that of non-ventricular arrhythmia group, which might be due to the increase of cardiac load caused by the change of hemodynamics affected by ventricular arrhythmia.

ECG is a technique for recording changes in cardiac electrical activity using an electrocardiograph. It is non-invasive, convenient and inexpensive. It is the main detection method for evaluating HF. This study revealed that common ECG indicators were correlated with BNP and PIIINP proteins, which suggested that BNP and PIIINP levels could be predicted. However, ROC curve analysis of BNP and PIIINP in this study indicated that BNP and PIIINP had diagnostic value for CHF, and could predict it in advance in combination with ECG indexes.

In conclusion, ECG indicators were correlated with BNP and PIIINP, and they showed diagnostic value for CHF. Using 
the indexes to predict BNP and PIIINP levels was conducive to the diagnosis of $\mathrm{CHF}$.

\section{Acknowledgements}

Not applicable.

\section{Funding}

No funding was received.

\section{Availability of data and materials}

The datasets used and/or analyzed during the current study are available from the corresponding author on reasonable request.

\section{Authors' contributions}

YW wrote the manuscript, interpreted and analyzed the data. XM designed the study and performed the experiments. YW was responsible for the analysis and discussion of the data. Both authors read and approved the final manuscript.

\section{Ethics approval and consent to participate}

The study was approved by the Ethics Committee of Yantai Yuhuangding Hospital (Yantai, China). Patients who participated in this research had complete clinical data. Signed informed consents were obtained from the patients and/or the guardians.

\section{Patient consent for publication}

Not applicable.

\section{Competing interests}

The authors declare that they have no competing interests.

\section{References}

1. Morrissey RP, Czer L and Shah PK: Chronic heart failure. Wiley Handbook of Current and Emerging Drug Therapies. Am J Cardiovasc Drugs 11: 153-171, 2006.

2. Hunt SA, Baker DW, Chin MH, Cinquegrani MP, Feldman AM, Francis GS, Ganiats TG, Goldstein S, Gregoratos G,JessupML, etal; American College of Cardiology/American Heart Association: ACC/AHA guidelines for the evaluation and management of chronic heart failure in the adult: Executive summary. A report of the American College of Cardiology/American Heart Association Task Force on Practice Guidelines (Committee to revise the 1995 Guidelines for the Evaluation and Management of Heart Failure). J Am Coll Cardiol 38: 2101-2113, 2001.

3. Hunt SA, Abraham WT, Chin MH, Feldman AM, Francis GS, Ganiats TG, Jessup M, Konstam MA, Mancini DM, Michl K, et al: ACC/AHA 2005 Guideline Update for the Diagnosis and Management of Chronic Heart Failure in the Adult-Summary Article: A Report of the American College of Cardiology/American Heart Association Task Force on Practice Guidelines (Writing Committee to Updat). J Am Coll Cardiol 46: 1116-1143, 2005.
4. Pocock SJ, Wang D, Pfeffer MA, Yusuf S, McMurray JJ Swedberg KB, Ostergren J, Michelson EL, Pieper KS and Granger CB: Predictors of mortality and morbidity in patients with chronic heart failure. Eur Heart J 27: 65-75, 2006.

5. Hendry PB, Krisdinarti L and Erika M: Scoring system based on electrocardiogram features to predict the type of heart failure in patients with chronic heart failure. Cardiol Res 7: 110-116, 2016.

6. Petrovic D: Cytopathological basis of heart failure - cardiomyocyte apoptosis, interstitial fibrosis and inflammatory cell response. Folia Biol (Praha) 50: 58-62, 2004.

7. Lepojärvi ES, Piira OP, Pääkkö E, Lammentausta E, Risteli J, Miettinen JA, Perkiömäki JS, Huikuri HV and Junttila MJ: Serum PINP, PIIINP, galectin-3, and ST2 as surrogates of myocardial fibrosis and echocardiographic left venticular diastolic filling properties. Front Physiol 6: 200, 2015.

8. Mukoyama M, Nakao K, Hosoda K, Suga S, Saito Y, Ogawa Y, Shirakami G, Jougasaki M, Obata K and Yasue H: Brain natriuretic peptide as a novel cardiac hormone in humans. Evidence for an exquisite dual natriuretic peptide system, atrial natriuretic peptide and brain natriuretic peptide. J Clin Invest 87: 1402-1412, 1991.

9. Anker SD, Coats AJ, Bonarjee VV, Caidahl K, Lie RT, Nilsen DW, Sundsfjord JA and Dickstein K: Plasma brain natriuretic peptide as an indicator of left ventricular systolic function and long-term survival after acute myocardial infarction. Circulation 95: 538-539, 1997.

10. Nagaya N, Nishikimi T, Uematsu M, Satoh T, Kyotani S, Sakamaki F, Kakishita M, Fukushima K, Okano Y, Nakanishi N, et al: Plasma brain natriuretic peptide as a prognostic indicator in patients with primary pulmonary hypertension. J Cardiol 37: 110-111, 2001 (In Japanese).

11. Degani R: Computerized electrocardiogram diagnosis: Fuzzy approach. Methods Inf Med 31: 225-233, 1992.

12. Nahler MPG: New York Heart Association classification (NYHA). In: Dictionary of Pharmaceutical Medicine. Springer, Vienna, pp121-121, 2009.

13. Wang Y, Xie B, Wan F, Xiao Q and Dai L: Application of ROC curve analysis in evaluating the performance of alien species potential distribution models. Biodiversity Sci 15: 365-372, 2007 (In Chinese).

14. Virk IS and Ip JR: The effect of cardiac resynchronization on morbidity and mortality in heart failure. Digest of the World Core Medical Journals 2005. https://doi.org/10.1111/ j.1527-5299.2005.04176.x. Accessed October 9, 2008.

15. Devereux RB and Reichek N: Echocardiographic determination of left ventricular mass in man. Anatomic validation of the method. Circulation 55: 613-618, 1977.

16. Droege ME, Mueller EW, Besl KM, Lemmink JA, Kramer EA, Athota KP, Droege CA, Ernst NE, Keegan SP, Lutomski DM, et al: Effect of a dalteparin prophylaxis protocol using anti-factor Xa concentrations on venous thromboembolism in high-risk trauma patients. J Trauma Acute Care Surg 76: 450-456, 2014.

17. Moe GW: BNP in the diagnosis and risk stratification of heart failure. Heart Fail Monit 4: 116-122, 2005.

18. Aldamiz-Echevarría Iraúrgui B, Muñiz J, Rodríguez-Fernández JA, Vidán-Martínez L, Silva-César M, Lamelo-Alfonsín F, Díaz-DíazJL, Ramos-Polledo V and Castro-Beiras A: Randomized controlled clinical trial of a home care unit intervention to reduce readmission and death rates in patients discharged from hospital following admission for heart failure. Rev Esp Cardiol 60: 914-922, 2007 (In Spanish).

19. Yonezawa E, Matsumoto K, Ueno K, Tachibana M, Hashimoto H, Komamura K, Kamakura S, Miyatake K and Tanaka K: Lack of interaction between amiodarone and mexiletine in cardiac arrhythmia patients. J Clin Pharmacol 42: 342-346, 2002.

This work is licensed under a Creative Commons Attribution-NonCommercial-NoDerivatives 4.0 International (CC BY-NC-ND 4.0) License. 